\title{
A Mean Value Model for Unsteady Gas Flows and Heat Transfer in Pipes
}

\author{
Olov Holmer Lars Eriksson \\ Vehicular Systems, Department of Electrical Engineering, Linköping University, Sweden \\ \{olov.holmer, lars.eriksson\} aliu.se
}

\begin{abstract}
Pipes are essential components in engines and therefore models of them are important. For example, the aftertreatment system for modern heavy-duty diesel engines consists of multiple components that are connected using pipes. The temperature in each of these components are important when determining the efficiency of the aftertreatment system and therefore models that accurately describe the temperature in the pipes between the components are important. Here, a dynamic pipe model that combines the adiabatic model of a control volume and that of a stationary one-dimensional flow with heat transfer in a pipe is developed and validated. The resulting model is a quasi-dimensional lumped parameter mean value model containing states for the temperature and pressure of the gas inside the pipe and the temperature of the pipe wall. The model uses the states and convective heat transfer models to calculate pressure at the inlet and outlet as well as temperature at the outlet, in a way that is physically correct under certain conditions. To validate the physical behavior of the model a detailed one-dimensional model is used, and to validate the practical applicability and accuracy of the model data from a passenger car gasoline engine is used to parameterize and validate the model.

Keywords: Pipe flow, Engine modeling, Heat transfer
\end{abstract}

\section{Introduction}

The complexity of modern powertrains are constantly increasing to meet the demands on better fuel efficiency and driveability while at the same time keep the emissions within the legislated levels. To achieve this, control systems that utilizes the complexity of the driveline are important. When designing control systems, especially for complex systems, models of the system can be very helpful when analyzing the problem as well as for speeding up testing procedures.

In engines, pipes are used to transport gas between the various components in the engine and therefore models of the are important as subcomponents in a larger model. For example, the gas conditions in the pipe connecting the engine with the aftertreatment system are important when determining the efficiency of the aftertreatment system. The efficiency of the aftertreatment system is most affected by the temperature, or energy, of the system.
There are two types of important processes, one is the heat transfer that occurs in a distributed manner along the flow and the other is compression and expansion of the gas inside the pipe. Currently there are accurate but complex distributed parameter models, GT-Power (Gamma Technologies, 2004), (Spring, 2006), etc. and there are compact control volume models for the gas compression and expansion (Hendricks, 2001; Eriksson and Nielsen, 2014) and there is a need for a compact model that can describe gas flow transients and heat transfer in pipe systems. Therefore, in this paper, a model that combines the adiabatic model of a control volume and that of a stationary one-dimensional flow with heat transfer, in a way that is energy consistent, is developed.

\subsection{Method}

The model is developed by combining a model for stationary one-dimensional flow from (Eriksson, 2002) with an adiabatic control volume described in (Eriksson and Nielsen, 2014). When combining the models, the different energy flows in a pipe are considered so that the energy inside the pipe is consistent with the energy flows in and out of the pipe.

To validate the model, measurements from a passenger car gasoline engine in a test stand as well as a detailed one-dimensional model are used.

\subsection{Contributions}

The main contribution is a new mean value model of a pipe and validation of this model using a detailed onedimensional model and measurements from an engine.

\subsection{Outline of the Paper}

The paper is organized as follows: In Section 2 the different heat transfer modes in a pipe are discussed, in Section 3 a detailed one-dimensional model of a pipe is presented, in Section 4 a new mean value model is presented, in Section 5 validation of the new model is done, and finally in Section 6 conclusions are made.

\section{Heat Transfer in Pipes}

Heat transfer occurs in three different ways in a pipe: between the gas inside the pipe and the pipe wall, between the pipe wall and the surrounding air, and between the pipe and the components connected at the ends of the pipe. In 
this section the different heat transfer modes are presented.

\subsection{Gas to Wall Heat transfer}

The internal heat transfer between the gas and wall, with temperatures $T_{g}$ and $T_{w}$, respectively, is

$$
\dot{Q}_{e}=h_{c v, i} A_{i}\left(T_{g}-T_{w}\right)
$$

where $h_{c v, i}$ is the heat transfer coefficient. For fully developed turbulent flow the Nusselt number is often used in the following way

$$
N u=\frac{h_{c v, i} D_{i}}{\lambda}
$$

where $D_{i}$ is the inner diameter of the pipe and $\lambda$ is the conductivity of the gas. Many empirical relations for the Nusselt number have been presented in literature (Wendland, 1993; Chen, 1993; Zhao and Winterbone, 1993; Eriksson, 2002), here the same as in (Eriksson, 2002) is used:

$$
N u=0.48 R e^{0.5}
$$

where $R e$ is the Reynolds number, which for a pipe is

$$
R e=\frac{4 W}{\pi D \mu} .
$$

\subsection{Wall to Ambient Heat Transfer}

The external heat transfer from the wall to the ambient air consist both of convection an radiation in the following way

$$
\dot{Q}_{e}=A_{e}\left(h_{c v, e}\left(T_{w}-T_{a m b}\right)+F_{v} \varepsilon \sigma\left(T_{w}^{4}-T_{a m b}^{4}\right)\right)
$$

where $F_{v}$ is the viewing factor, $\varepsilon$ is the emissivity, $\sigma$ is the Stefan-Boltzmann constant, and $h_{c v, e}$ is the convective heat transfer coefficient. Since the engine is mounted in a test stand, where the ambient air is standing still, there is only natural convection. For natural convection the following relationship, from (Eastop and Mc Conkey, 1986), is used

$$
h_{c v, e}= \begin{cases}1.33\left(\frac{T_{w}-T_{a m b}}{D_{e}}\right)^{1 / 4}, & 10^{4}<G r \leq 10^{9} \\ 1.25\left(T_{w}-T_{a m b}\right)^{1 / 3}, & 10^{9}<G r<10^{12}\end{cases}
$$

where $G r$ is the Grasshof number. In this case the Grasshof number is calculated using

$$
G r=\frac{\beta g D_{e}^{3}\left(T_{w}-T_{a m b}\right)}{v^{2}}
$$

where $\beta$ is the volume expansion coefficient, $g$ is the gravitational acceleration, and $v$ is the kinematic viscosity of the fluid.

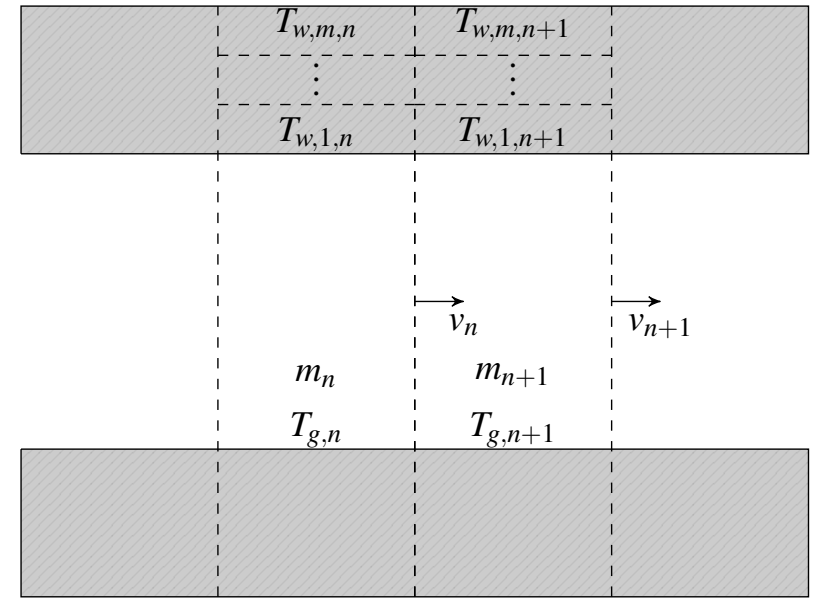

Figure 1. Illustration of how the pipe is split into segments in the one-dimensional model, some of the notation is also shown.

\subsection{Heat Conduction in the Pipe Wall}

Conduction occurs both radially and axially in the pipe wall. The radial conduction between two wall segments with length $L_{s}$ diameter $D_{1}$ and $D_{2}$, and temperatures $T_{w, 1}$ and $T_{w, 2}$ is (Holman, 1986)

$$
\dot{Q}_{\text {rad }}=\frac{2 \pi \lambda L_{s}}{\ln \left(\frac{D_{2}}{D_{1}}\right)}\left(T_{w, 1}-T_{w, 2}\right)
$$

where $\lambda$ is the conductivity of the wall material.

The axial conduction between two wall segment at distance $L_{s}$ of each other and temperatures $T_{w, 1}$ and $T_{w, 2}$ is

$$
\dot{Q}_{a x}=\frac{\lambda}{L_{s}} A_{c}\left(T_{w, 1}-T_{w, 2}\right)
$$

where $A_{c}$ is the cross section area between the segments.

\section{One-Dimensional Model}

In this section a detailed one-dimensional model is presented. In this model the pipe is split lengthwise into $N$ segments. Each segment contains states for the temperature of the gas inside the segment $T_{g, n}$, mass of the gas, $m_{n}$, and the velocity of the gas flowing to the next segment, $v_{n}$. The pipe wall in the segment is split into $M$ smaller segments with their own temperature, giving $M$ states for the temperature of the pipe wall. An illustration of how the pipe is split into segments is shown in Figure 1, where also some of the notation is shown.

\subsection{Mass Balance and Flow}

In each segment, the ideal gas law

$$
p_{n} V_{s}=m_{n} R T_{g, n}
$$

gives us the pressure

$$
p_{n}=\frac{m_{n} R T_{g, n}}{V_{s}}
$$


and the density of the gas

$$
\rho_{n}=\frac{m_{n}}{V_{s}}=\frac{p_{n}}{R T_{g, n}} .
$$

The mass flow from segment $n$ to $n+1$ is calculated by considering a plug moving from segment $n$ to $n+1$. The weight of the plug is

$$
m_{v, n}=\frac{m_{n}+m_{n+1}}{2}
$$

and the velocity, $v_{n}$, of the plug is given by

$$
m_{v, n} \frac{d v_{n}}{d t}=A_{c}\left(p_{n}-p_{n+1}-\Delta p_{f}\left(v_{n}\right)\right)
$$

where $A_{c}$ is the cross section area of the pipe channel and $\Delta p_{f}(v)$ is the pressure drop due to friction in the pipe. In (Cengel et al., 2008) the relation for the pressure drop is given as

$$
\Delta p_{f}(v)=f(R e) \rho v^{2} \frac{L_{s}}{2 D} .
$$

where $f$ is the friction factor given by the Colebrook equation

$$
\frac{1}{\sqrt{f}}=-2 \log _{10}\left(\frac{\varepsilon / D}{3.7}+\frac{2.51}{\operatorname{Re} \sqrt{f}}\right)
$$

which can be approximated well with the following closed form expression

$$
\frac{1}{\sqrt{f}} \approx-1.8 \log _{10}\left(\frac{6.9}{\operatorname{Re}}+\left(\frac{\varepsilon / D}{3.7}\right)^{1.11}\right) .
$$

Finally, the mass flow from segment $n$ to $n+1$ can be calculated as

$$
W_{n}=A_{c} \rho_{n} v_{n} .
$$

\subsection{Gas Temperature}

The dynamics of the gas temperature is

$$
\begin{aligned}
c_{v} m_{g, n} \frac{d T_{g, n}}{d t}= & W_{n-1}\left(c_{v}\left(T_{p, n-1}-T_{g, n}\right)+R T_{p, n-1}\right) \\
& -W_{n}\left(c_{v}\left(T_{p, n}-T_{g, n}\right)+R T_{p, n}\right)+\dot{Q}_{i}
\end{aligned}
$$

where

$$
T_{p, n}= \begin{cases}T_{g, n}, & w_{n-1} \geq 0 \\ T_{g, n+1}, & w_{n-1}<0\end{cases}
$$

is the temperature of the moving plug.

\subsection{Wall Temperature}

The differential equations for the wall segments depend on the position of the segment. For the wall segments on the inner layer $(m=1)$ the equation is

$$
\begin{aligned}
\frac{d T_{w, 1, n}}{d t}= & h_{c v, i, n}\left(T_{g, n}-T_{w, 1, n}\right) \\
& +\dot{Q}_{a x}\left(T_{w, 1, n+1}, T_{w, 1, n}\right) \\
& +\dot{Q}_{a x}\left(T_{w, 1, n-1}, T_{w, 1, n}\right) \\
& +\dot{Q}_{a a d}\left(T_{w, 2, n}, T_{w, 1, n}\right)
\end{aligned}
$$

for segments on the outer layer $(m=M)$

$$
\begin{aligned}
\frac{d T_{w, 1, n}}{d t}= & h_{c v, e, n}\left(T_{a m b}-T_{w, 1, n}\right) \\
& +\dot{Q}_{a x}\left(T_{w, M, n+1}, T_{w, M, n}\right) \\
& +\dot{Q}_{a x}\left(T_{w, M, n-1}, T_{w, M, n}\right) \\
& +\dot{Q}_{r a d}\left(T_{w, M-1, n}, T_{w, M, n}\right)
\end{aligned}
$$

and for segments that are fully inside the pipe

$$
\begin{aligned}
\frac{d T_{w, m, n}}{d t}= & \dot{Q}_{a x}\left(T_{w, m, n+1}, T_{w, m, n}\right) \\
& +\dot{Q}_{a x}\left(T_{w, m, n-1}, T_{w, m, n}\right) \\
& +\dot{Q}_{r a d}\left(T_{w, m+1, n}, T_{w, m, n}\right) \\
& +\dot{Q}_{r a d}\left(T_{w, m-1, n}, T_{w, m, n}\right) .
\end{aligned}
$$

\section{Mean Value Model}

The mean value model combines a model of an adiabatic control volume with stationary heat transfer. The model contains three states: wall temperature, $T_{w}$, mean gas temperature, $T_{m}$, and the mass of the gas inside the pipe, $m_{g}$. In this section the details of the model are presented.

\subsection{Wall Temperature}

The dynamics of the pipe wall temperature is

$$
c_{w} m_{w} \frac{d T_{w}}{d t}=\dot{Q}_{i}+\dot{Q}_{e}
$$

where

$$
\dot{Q}_{e, n}=A_{o}\left(h_{c v, e}\left(T_{w}-T_{a m b}\right)+F_{v} \varepsilon \sigma\left(T_{w}^{4}-T_{a m b}^{4}\right)\right)
$$

is the external heat transfer, and

$$
\dot{Q}_{i, n}=h_{c v, i} A_{i}\left(T_{m}-T_{w}\right)
$$

is the internal heat transfer.

\subsection{Mean Gas Temperature}

The change of energy of the gas inside the pipe, if we consider the pipe as an adiabatic control volume (Eriksson and Nielsen, 2014), is

$$
\dot{Q}=c_{v}\left(W_{\text {in }} T_{\text {in }}-T_{m}\right)+R\left(T_{\text {in }} W_{\text {in }}-W_{\text {out }} T_{\text {out }}\right)-\dot{Q}_{i}
$$

and this means that the change in mean temperature, $T_{m}$, can be calculated as

$$
c_{p} m_{g} \frac{d T_{m}}{d t}=c_{v}\left(W_{\text {in }} T_{\text {in }}-T_{m}\right)+R\left(T_{\text {in }} W_{\text {in }}-W_{\text {out }} T_{\text {out }}\right)-\dot{Q}_{i}
$$

\subsection{Mass Balance}

The mass of the gas in the pipe $m_{g}$ is calculated using

$$
\frac{d m_{g}}{d t}=W_{\text {in }}-W_{\text {out }}
$$

where $W_{\text {in }}$ and $W_{\text {out }}$ is the mass flow in and out of the pipe, respectively. 


\subsection{Temperature Drop Along the Pipe}

During stationary conditions, the temperature of the gas at position $l$ down the pipe is (Eriksson, 2002)

$$
T(l)=T_{w}+\Delta T e^{-k l}
$$

where $\Delta T=T_{i n}-T_{w}$ and

$$
k=\frac{\pi D_{i} h_{c v, i}}{W c_{p}}
$$

here, because of the stationary conditions, $W=W_{\text {in }}=$ $W_{\text {out }}$. During non-stationary conditions, however, $T(l)$ can essentially be arbitrary, depending on previous conditions, under the condition that the energy in the pipe must be consistent i.e.

$$
c_{p} m_{g} T_{m}=\int_{0}^{L} c_{p} \frac{m_{g}}{L} T(l) d l
$$

The assumption that $T(l)$ have the same exponential structure as in (30), but with a $\Delta T$ that makes (32) hold, results in

$$
\Delta T=\frac{k L\left(T_{m}-T_{w}\right)}{1-e^{-k L}} .
$$

During non-stationary conditions it does not hold that $W=$ $W_{\text {in }}=W_{\text {out }}$ and instead $W=\frac{W_{\text {in }}+W_{\text {out }}}{2}$ is used.

\subsection{Transport Delay}

The fact that the gas moves with a velocity

$$
v=\frac{W}{\rho A_{i}}=\frac{W R T}{p A_{i}}
$$

in a pipe of length $L$ gives rise to a transport delay

$$
\tau_{d}=\frac{L}{v}=\frac{L A_{i} p}{W R T}=/ \begin{gathered}
L A_{i}=V \\
p V=m R T
\end{gathered} /=\frac{m}{W} .
$$

from the inlet to the outlet. This transport delay can be included in the model by using a time delayed version of $T_{m}$ when calculating the output temperature.

\section{Validation and Comparison}

In this section the new mean value model is validated in three different ways: fist the stationary cooling of the gas is validated using stationary measurements, then the gas mixing dynamics are validated using the one-dimensional model, and finally the model is validated using dynamic measurements from an engine. The engine on which the measurements were made is a turbocharged 2 liter inline 4-cylinder gasoline engine. The measurements in this paper have been done on the pipe between the compressor and the intercooler. This is a pipe that is approximately $2 \mathrm{~m}$ long, has a diameter of approximately $5 \mathrm{~cm}$, and is made of rubber and plastics. The pipe is also equipped with temperature and pressure sensors at both ends.
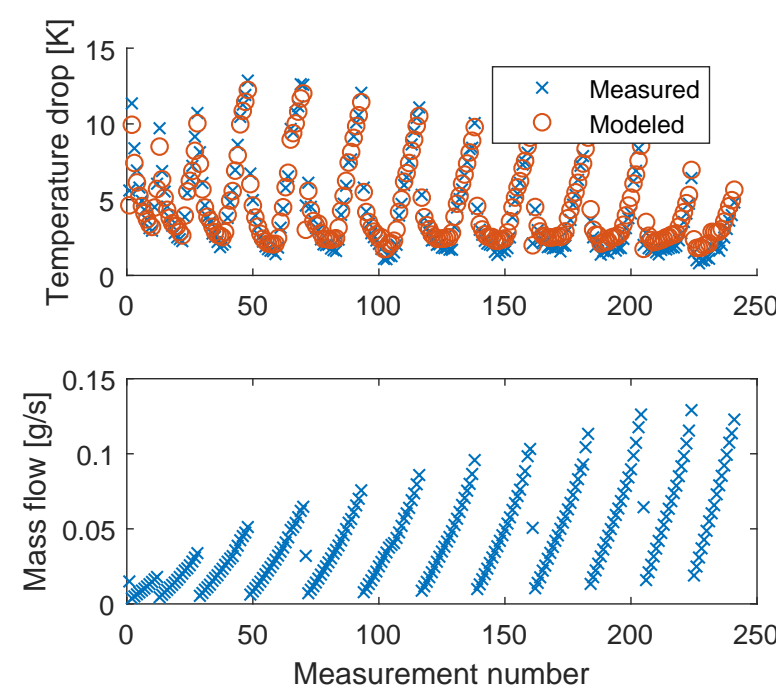

Figure 2. Measured and modeled stationary temperature drop between the compressor and intercooler. The upper figure shows the temperature drop and the lower shows the mass flow.

\subsection{Stationary Cooling}

In (Eriksson, 2002) the stationary cooling for exhaust pipes are validated and the principles for a pipe on the intake side are the same as on the exhaust pipes. However, the assumption of constant wall temperature might be less accurate since the pipes are made out of different materials with less conductivity. Therefore the model is here validated for pipes on the intake side of the engine. In Figure 2 the temperature drop in the pipe between the compressor and intercooler is shown for different mass flows. During stationary conditions the mass flow is the same throughout the whole intake side and therefore the mass flow is taken directly from the mass flow sensor, which is mounted slightly before the compressor. As can be seen the model predicts the pressure drop well, so it can be concluded that the model performs well in stationary conditions and the assumption of constant wall temperature is a reasonable assumption on the intake side as well.

\subsection{Comparison with One-dimensional Model}

The gas mixing has a time constant in the order of seconds. This makes it hard to measure it with temperature sensors, since they have a time constant that is similar or larger. Therefore, to validate these dynamics, simulations from the one-dimensional model are used. Two different simulations were done, one were a step in input temperature was made and one were a step in the input flow was made.

In Figure 3 the result from the step in temperature is shown. The step is made after 10 seconds and as expected the big change in output is delayed since it takes some time for the new temperature to propagate through the pipe. However, the one-dimensional model shows a small rise in temperature directly after the step, this is because the pressure in the pipe increases with the temperature and 
this causes a temperature change that moves much quicker than the velocity of the gas, the same effect is present in the mean value model but is barely visible. It can also be seen that the mean temperature of the two models are the same before and after the step, but during the transient they differ. The reason for this is that the assumption of exponential decay of the temperature is not valid and therefore the heat transfer in the mean value model is not correct during transients, however, the difference is not that big.

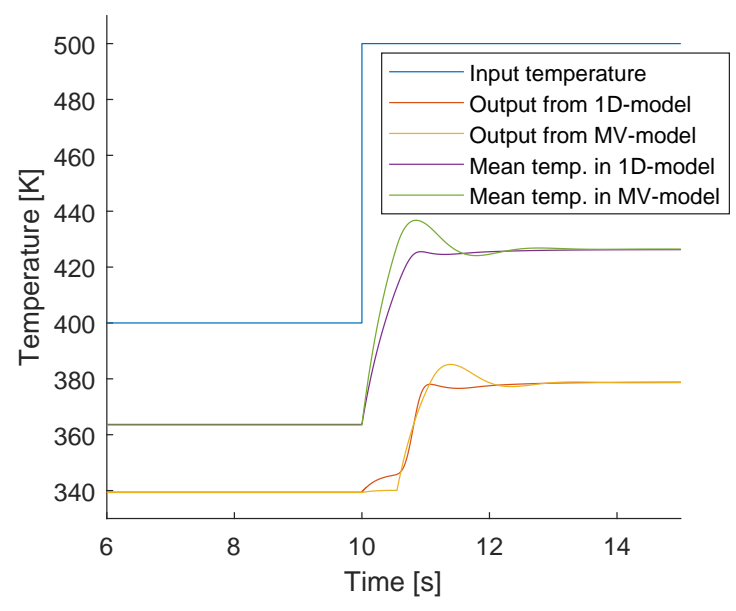

Figure 3. Output temperature and mean temperature from the one-dimensional and mean value model when a step is made in the input temperature.

Figure 4 shows the result when the step in input flow is made. Here it again can be seen that the temperature rises without a time delay, and again this is because the change in pressure is not affected by the transport delay. Here it is more evident that the mean value model does not describe this well, there is a small increase in temperature at the time of the step but most of the temperature change is delayed in the mean value model. The small initial increase in temperature in the mean value model is because of that the mass flow used to calculate the heat transfer along the pipe is the mean of the input and output flow, and since the input flow increases the mean flow increases which reduces the heat transfer to the pipe wall.

In the two examples above a step was used as input signal and that type of drastic change really tests the dynamic behavior of the model. Since this type of drastic change are not likely to occur in an engine, this could be considered to be outside the region where the mean value model is designed to be used. In Figure 5 the same step in temperature as in Figure 3, but now filtered with a time constant of $1 \mathrm{~s}$, is used as input to the models. When using this input, with a smaller rate of change, it can be seen that the agreement between the models is better.

\subsection{Dynamic Measurements}

Here dynamic measurements where a step in wastegate position was made to increase the mass flow through the

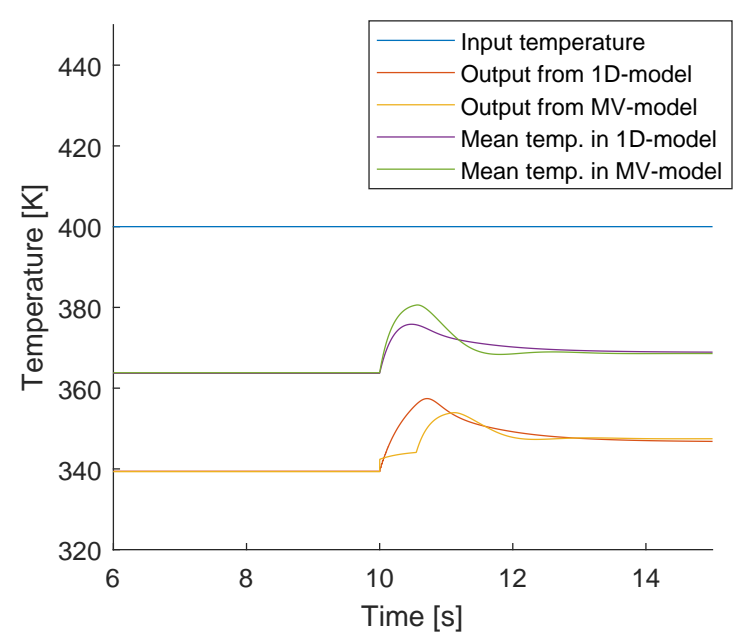

Figure 4. Output temperature and mean temperature from the one-dimensional and mean value model when a step is made in the input flow.

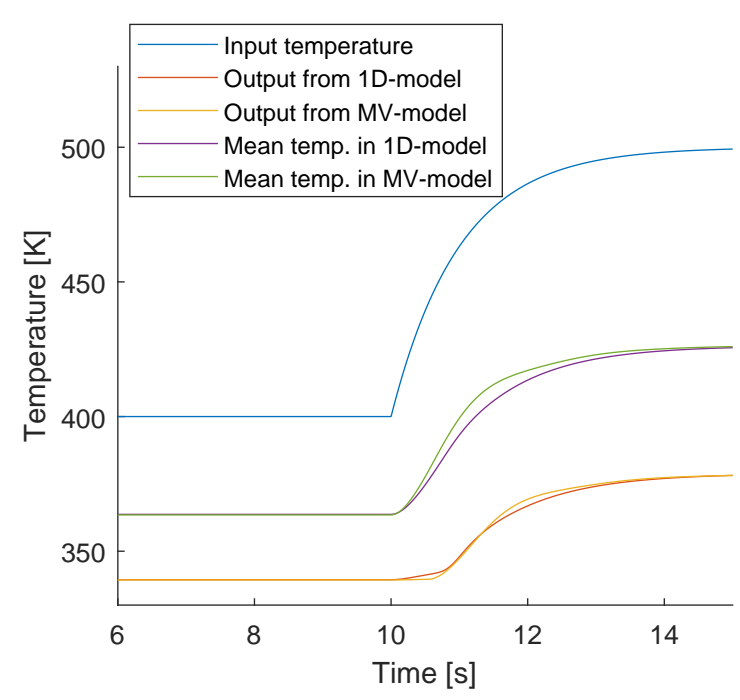

Figure 5. Output temperature and mean temperature from the one-dimensional and mean value model when the input temperature is a step filtered with a time constant of $1 \mathrm{~s}$.

compressor and increase the temperature after the compressor are used to validate the model. The temperature after the compressor was used as input to the model and the temperature before the intercooler was used as validation data. During transients the mass flow sensor can not be used directly since it is mounted at some distance from the pipe. Therefore the mass flow sensor was only used to determine the stationary levels before and after the step, during the step the mass flow in and out of the pipe was assumed to move between these two levels in the following way: the input flow was assumed to increase linearly with the compressor angular velocity and the output flow was assumed to increase linearly with the pressure drop over the intercooler.

It was also discovered that there were some additional 
dynamics, with a time constant of around $15 \mathrm{~s}$, present in the measurements. These additional dynamics could come from the dynamics of the temperature sensor. However, the time constant might be somewhat too big to be explained solely by this, therefore further investigation is needed to investigate this phenomenon.

In Figure 6 the measured and modeled output when the sensor dynamics have been included by filtering the output from the model with the time constant of sensor is shown. As can be seen the model seems to agrees well with the measurements.

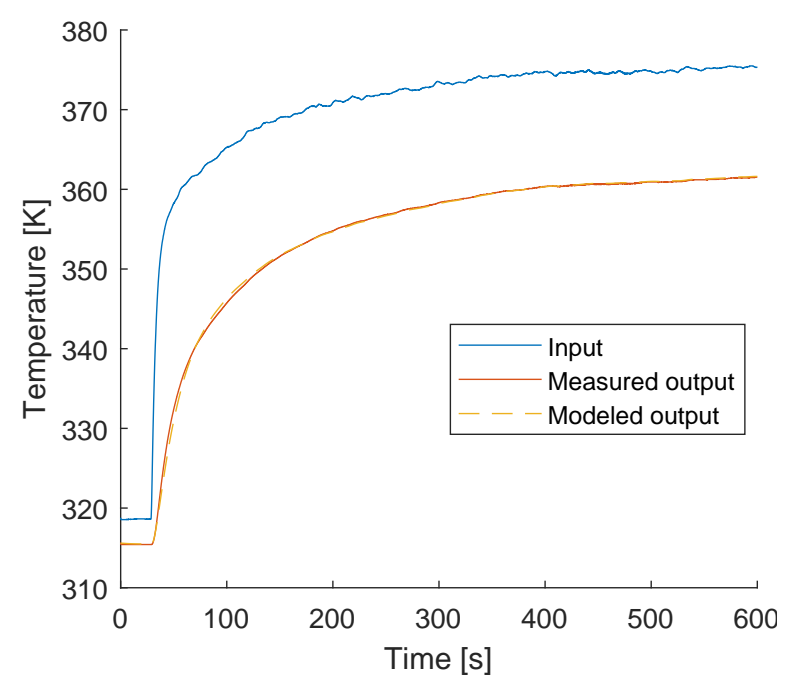

Figure 6. Dynamic measurements from the engine and the output from the model. Here the dynamics of the sensor is included by filtering the output from the model with a time constant of $15 \mathrm{~s}$.

Figure 7 shows the same test as in Figure 6, but now one simulation without the wall temperature dynamics and one without the sensor dynamics is also shown. Here it becomes clear that both these dynamics are needed to capture the dynamics of the system, the sensor dynamics are needed to reduce the initial rate of change and the wall temperature dynamics are needed to capture the slower rate of change towards the stationary level.

\section{Conclusions}

A dynamic pipe model that combines the adiabatic model of a control volume and that of a stationary onedimensional flow with heat transfer in a pipe has been developed and validated. The validation has been done using both measurements from an engine in a test stand as well as simulations from a detailed one-dimensional model. The model has shown to agree well with the measurements from the engine and the simulations from the one-dimensional model.

\subsection{Future Work}

Interesting future work would be to try to separate temperature changes in the inlet temperature from changes in

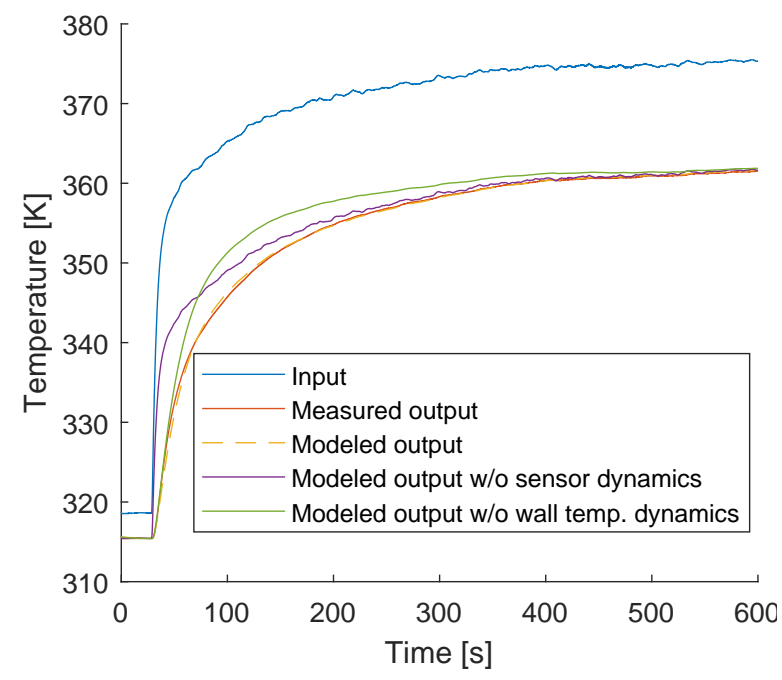

Figure 7. Dynamic measurements from the engine and the output from the model. Here the output with and without the sensor dynamics are shown as well as the output when the wall temperature dynamics are removed.

temperature due to compression of the gas, since temperature changes from compression are not affected by the transport delay that changes in inlet temperature are affected by.

\section{References}

Yunus A Cengel, Robert H Turner, John M Cimbala, and Mehmet Kanoglu. Fundamentals of thermal-fluid sciences. McGraw-Hill New York, NY, 2008.

David KS Chen. A numerical model for thermal problems in exhaust systems. Technical report, SAE Technical Paper, 1993.

Thomas D Eastop and Allan Mc Conkey. Applied thermodynamics for engineering technologies. 1986.

Lars Eriksson. Mean value models for exhaust system temperatures. Technical report, SAE Technical Paper, 2002.

Lars Eriksson and Lars Nielsen. Modeling and control of engines and drivelines. John Wiley \& Sons, 2014.

Gamma Technologies. GT-Power User's Manual. GT-Suite Version 6.1, 2004.

Elbert Hendricks. Isothermal vs. adiabatic mean value si engine models. IFAC Proceedings Volumes, 34(1):363-368, 2001.

JP Holman. Heat transfer, 1986. Mc Gran-Hill Book Company, Soythern Methodist University, 1986.

Peter Spring. Modeling and control of pressure-wave supercharged engine systems. ETH Zurich, 2006.

Daniel W Wendland. Automobile exhaust-system steady-state heat transfer. Technical report, SAE Technical Paper, 1993.

Y Zhao and DE Winterbone. A study of warm-up processes in si engine exhaust systems. Technical report, SAE Technical Paper, 1993. 